\title{
Sublinear Algorithms in T-interval Dynamic Networks
}

\author{
Irvan Jahja* \\ National University of Singapore \\ Republic of Singapore \\ irvan@comp.nus.edu.sg
}

\author{
Haifeng $\mathrm{Yu}^{*}$ \\ National University of Singapore \\ Republic of Singapore \\ haifeng@comp.nus.edu.sg
}

\begin{abstract}
We consider standard T-interval dynamic networks, under the synchronous timing model and the broadcast CONGEST model. In a T-interval dynamic network, the set of nodes is always fixed and there are no node failures. The edges in the network are always undirected, but the set of edges in the topology may change arbitrarily from round to round, as determined by some adversary and subject to the following constraint: For every $T$ consecutive rounds, the topologies in those rounds must contain a common connected spanning subgraph. Let $H_{r}$ to be the maximum (in terms of number of edges) such subgraph for round $r$ through $r+T-1$. We define the backbone diameter $d$ of a $T$-interval dynamic network to be the maximum diameter of all such $H_{r}$ 's, for $r \geq 1$. We use $n$ to denote the number of nodes in the network.

Within such a context, we consider a range of fundamental distributed computing problems including Count/MAX/MEDIAN/Sum/ LEADERElect/Consensus/ConfirmedFlood. Existing algorithms for these problems all have time complexity of $\Omega(n)$ rounds, even for $T=\infty$ and even when $d$ is as small as $O(1)$. This paper presents a novel $O\left(d^{3} \log ^{2} n\right)$ deterministic algorithm for computing CounT, for $T$-interval dynamic networks with $T \geq c \cdot d^{2} \log ^{2} n$. Here $c$ is a (sufficiently large) constant independent of $d, n$, and $T$. To our knowledge, our algorithm is the very first such algorithm whose complexity does not contain a $\Theta(n)$ term. For $d=O\left(n^{a}\right)$ with constant $a<\frac{1}{3}$, our deterministic algorithm has $o(n)$ complexity, which is better than all (both randomized and deterministic) existing Count algorithms in this setting. For $d=O(\operatorname{polylog}(n))$, our algorithm is exponentially faster. Following the framework of our Count algorithm, this paper further develops novel algorithms for solving MAX/Median/Sum/LEAdERELECT/ CONSENSUS/CONFIRmedFlood, while incurring either $O\left(d^{3} \log ^{2} n\right)$ or $O\left(d^{3} \log ^{3} n\right)$ complexity. Again, for all these problems, our algorithms are the first ones whose time complexity does not contain a $\Theta(n)$ term.
\end{abstract}

\section{CCS CONCEPTS}

\section{- Theory of computation $\rightarrow$ Distributed algorithms.}

"The authors of this paper are alphabetically ordered.

Permission to make digital or hard copies of part or all of this work for personal or classroom use is granted without fee provided that copies are not made or distributed for profit or commercial advantage and that copies bear this notice and the full citation on the first page. Copyrights for third-party components of this work must be honored. For all other uses, contact the owner/author(s)

SPAA'20, fuly 15-17, 2020, Virtual Event, USA

(C) 2020 Copyright held by the owner/author(s).

ACM ISBN 978-1-4503-6935-0/20/07.

https://doi.org/10.1145/3350755.3400228

\section{KEYWORDS}

distributed algorithms; $T$-interval dynamic networks; sublinear algorithms

\section{ACM Reference Format:}

Irvan Jahja and Haifeng Yu. 2020. Sublinear Algorithms in T-interval Dynamic Networks. In Proceedings of the 32nd ACM Symposium on Parallelism in Algorithms and Architectures (SPAA'20), fuly 15-17, 2020, Virtual Event, USA. ACM, New York, NY, USA, 11 pages. https://doi.org/10.1145/3350755. 3400228

\section{INTRODUCTION}

Our setting. We consider various fundamental distributed computing problems in standard T-interval dynamic networks $[1,2,6$, $11,13,18]$, under the synchronous timing model. The network has a fixed set of $n$ nodes, which proceed in lock-step rounds, starting from round 1. Each node has a unique id of $O(\log n)$ size. The algorithm knows neither $n$ nor any upper bound on $n .{ }^{1}$ In a T-interval dynamic network $(T \geq 1)$, the edges are always undirected, but the set of edges in the topology may change arbitrarily from round to round, as determined by some adversary and subject to the following constraint: For every $T$ consecutive rounds, the topologies in those rounds must contain a common connected spanning subgraph, which implies that this subgraph remains stable in those $T$ rounds. (Note that this subgraph is required to be both connected and spanning.) Let $H_{r}$ to be the maximum (in terms of number of edges) such subgraph for the $T$ rounds from round $r$ to $r+T-1$. We define the backbone diameter $d$ of a $T$-interval dynamic network to be the maximum diameter of all such $H_{r}$ 's, for $r \geq 1$. The distributed algorithm knows neither $d$ nor any upper bound on $d$. The above notions can also be extended to $T=\infty$. Namely, in an $\infty$-interval dynamic network, the adversary guarantees that the topologies in all rounds contain a common connected spanning subgraph. Let $H$ be the maximum (in terms of number of edges) such subgraph. We define the backbone diameter $d$ of an $\infty$-interval dynamic network to be the diameter of this graph $H$. This paper will focus on $T$-interval dynamic networks with sufficiently large $T$ (see exact condition later), including $T=\infty$.

Following [1, 2, 13], we use the broadcast CONGEST model [27] where in each round, each node is allowed to choose a single message of $O(\log n)$ size, and send the message simultaneously to all its neighbors. (A node cannot send different messages to different neighbors.) Without loss of generality, we assume that a message always contains its sender's id. At the end of each round, each node receives all the messages sent in that round by all its neighbors (as determined by the topology of that round). Note that, a node

\footnotetext{
${ }^{1}$ As in $[1,2,11,13]$, we have assumed that each node has a unique id of size $O(\log n)$. This means the largest id among the $n$ nodes maps to a loose polynomial upper bound on $n$. However, finding the largest id among the $n$ nodes is at least as hard as the LEADERELECT problem (formally defined later), and hence is non-trivial by itself.
} 
does not know its neighbors, before it receives messages from them. Also, a node does not know the topology in each round.

The time complexity (or simply complexity) of a distributed algorithm for solving a certain problem is defined to be the number of rounds needed for all nodes to output and terminate, under the worst-case input and worst-case adversary ${ }^{2}$. We describe the time complexity as a function of $n$ and $d$. The central challenge in designing distributed algorithms in $T$-interval dynamic networks is that the $H_{r}$ 's (or $H$ ) and $d$ are all unknown to the algorithm, and that in each round, the algorithm does not know beforehand which edges in the network will survive and which edges will be deleted/added.

Problems and existing results. We consider the following fundamental distributed computing problems in $T$-interval dynamic networks, where each node has an input of $O(\log n)$ size:

- Count: All nodes should output $n$.

- MAx/MediAn/Sum: All nodes should output the max/median/ sum of the $n$ inputs (as integers).

- LeaderElect: A unique leader should be elected, and all nodes should output the leader's id.

- Consensus: All nodes should output some common consensus value, while satisfying the standard agreement, validity, and termination requirements [25].

- ConfirmedFlood [30]: One distinguished node needs to propagate its input to all nodes, and then should output " 1 " after all nodes have received its input.

We note that all these distributed computing problems are nontrivial to solve, even in $\infty$-interval dynamic networks, given that $d$ and $H$ are not known beforehand. ${ }^{3}$ To our knowledge, all existing deterministic and randomized ${ }^{4}$ algorithms $[1,6,11,13,18]$ for all the above problems have time complexity of $\Omega(n)$ rounds in $T$-interval dynamic networks, even for $T=\infty$. A dynamic network's backbone diameter $d$ may range from 1 to $n-1$. While the existing works $[1$, $6,11,13,18]$ do not describe their algorithms' time complexities in terms of both $n$ and $d$, it can be easily verified that their time complexities remain $\Omega(n)$ even when $d$ is as small as $O(1)$ (and even when $T=\infty$ ). Putting it another way, if these complexities were described as functions of both $n$ and $d$, such functions would still all be $\Omega(n)$. The reason for such $\Omega(n)$ complexity is that existing approaches usually solve these problems by each node collecting all $n$ inputs - namely, these problems are often solved as a byproduct of solving token dissemination $[1,6,11,13]$. But token dissemination fundamentally requires each node to receive $\Omega(n \log n)$ bits, which takes $\Omega(n)$ rounds for a constant degree node, even when $d=O(1)$.

Solving Count/Max/Median/Sum/LeaderElect/Consensus/ ConfirmedFlood by collecting all $n$ inputs appears to be quite an overkill. All these problems are "global" in the sense that the output could be affected by far away nodes in the network. Such

\footnotetext{
${ }^{2}$ We will mainly be concerned with deterministic algorithms, where it is irrelevant whether the adversary can see and then adapt to the coin flip outcomes in the algorithm. (Namely, it is irrelevant whether the adversary is oblivious or adaptive.)

${ }^{3}$ If $H$ is known, then regardless of whether $d$ is known, one can trivially solve all these problems in $O(d)$ rounds, by doing simple tree-based aggregation over edges in $H$. If $H$ is not known but $d$ is known, then MAX/LEADERELECT/CONSENSUS/CONFIRMEDFLOOD can all be solved trivially via flooding in $O(d)$ rounds, while Count/Median/Sum remain non-trivial. If neither $H$ nor $d$ is known, then all these problems are non-trivial ${ }^{4} \mathrm{~A}$ randomized algorithm is designed either for oblivious adversaries or adaptive adversaries. The complexity of a randomized algorithm is always defined under the worst-case adversary for which the algorithm is designed.
}

a need for "global" information does lead to an $\Omega(d)$ lower bound, but not an $\Omega(n)$ lower bound. While there is no hope of getting $o(n)$ complexity when $d=\Theta(n)$, it seems that we still should be able to solve these "global" problems in less than $n$ rounds when $d$ is small. Despite such natural thoughts, no existing algorithms can achieve this.

Our results. This paper first presents a novel deterministic CounT algorithm with $O\left(d^{3} \log ^{2} n\right)$ complexity, for $T$-interval dynamic networks with $T \geq c \cdot d^{2} \log ^{2} n$. Here $c$ is a (sufficiently large) constant independent of $d, n$, and $T$. (Throughout this paper, whenever we say $T$ is larger than some value, it always includes the limiting case of $T=\infty$.) To our knowledge, our algorithm is the very first such algorithm whose complexity does not contain a $\Theta(n)$ term. For $d=O\left(n^{a}\right)$ with constant $a<\frac{1}{3}$, our deterministic algorithm has $o(n)$ complexity, which is better than all existing (both deterministic and randomized) CounT algorithms in this setting. For $d=O(\operatorname{polylog}(n))$, our algorithm is exponentially faster than all existing algorithms.

Following the framework of our CounT algorithm, the paper further develops novel algorithms for solving MAX/MEDIAN/SuM/ LEAdERELECT/CONSENSUS/CONFIRMEDFlood, while incurring either $O\left(d^{3} \log ^{2} n\right)$ or $O\left(d^{3} \log ^{3} n\right)$ complexity, in $T$-interval dynamic networks with $T \geq c d^{2} \log ^{2} n$ for some (sufficiently large) constant $c$. Again, for all these problems, our algorithms are the first ones without a $\Theta(n)$ term in its complexity, achieving $o(n)$ complexity when $d=O\left(n^{a}\right)$ with constant $a<\frac{1}{3}$.

Finally, when $T<c d^{2} \log ^{2} n$ or when $d$ is large, our algorithms can no longer achieve $o(n)$ complexity. Obtaining sublinear algorithms under those parameter ranges will be our future work.

Implications of our results. For $T \geq c d^{2} \log ^{2} n$ and when $d=$ $O\left(n^{a}\right)$ with constant $a<\frac{1}{3}$, our new algorithms confirm that COUnT/MAX/MEdian/Sum/LeAderElect/CONSEnSus/CONFIRMEdFlood are indeed all easier than token dissemination (which has a lower bound of $\Omega(n)$ ). This implies that future research can benefit from approaching these problems directly, as compared to viewing their solutions as the byproduct of solving token dissemination $[1,6,11,13]$.

Our results have some further implications. It is known that even when $d=O(1)$, problems such as CounT and Sum have $\Omega(\operatorname{poly}(n))$ lower bounds in all the following settings:

- Same as our $\infty$-interval setting except that in each round, a node can choose to either send a message or receive messages, but cannot do both [30]. ${ }^{5}$

- Same as our $\infty$-interval setting except that the set of nodes (instead of the edges) can change (by crashing) from round to round [9].

- Same as our $\infty$-interval setting except that the topology is directed and never changes [20].

Despite some of the above settings being seemingly close to our setting, our $O\left(d^{3} \log ^{2} n\right)$ upper bound for Count and Sum implies that such $\Omega(\operatorname{poly}(n))$ lower bound can never carry over to our setting. The only currently known lower bound in our setting is the trivial $\Omega(d)$ lower bound.

\footnotetext{
${ }^{5}$ While [30] does not explicitly mention the $\infty$-interval model, its proofs apply without any change.
} 
Our approach. The approach taken by our algorithms is quite different from most existing approaches in dynamic networks for solving these problems. At the highest level, we rely on the classic idea of aggregation. In this classic approach, there is a rooted spanning tree and each node contributes a value of 1 . These values are propagated upstream along the tree paths to the root, while being aggregated (i.e., summed together) along the way. The root can then eventually learn the total count of nodes from the final sum. In dynamic networks however, such aggregation can be easily disrupted by the topology changes. To deal with this, conceptually, we do massively parallel aggregation simultaneously along many (up to exponential number of) aggregation paths. We further stagger the aggregation, together with carefully designed re-tries, to limit the adversary's damage. Next, we use a number of tricks (e.g., by allowing repeated nodes in an aggregation path), to minimize the amount of bookkeeping needed when dealing with a large number of aggregation paths - otherwise the design would have been highly inefficient. Finally, naively applying the previous ideas would require the knowledge of $n$ and $d$, as well as a unique leader node, which would beat the purpose. We use several techniques to overcome this.

More discussions on related works. As mentioned earlier, in the $T$-interval model, researchers often solves problems (such as CounT and LEADERELECT) that are functions of the $n$ inputs/ids, by having each node collect all the $n$ inputs/ids [1, 6, 11, 13, 18]. Collecting all the $n$ inputs/ids is also explicitly studied as the token dissemination problem. (Some of these works $[1,6,11,13,18]$ actually focus on token dissemination, while solving problems such as CounT as byproduct.) Kuhn et al. [18] have further explored solving CounT without collecting all $n$ inputs/ids. They propose an elegant randomized algorithm for computing a constant factor approximation for $n$ in $O(n \log \log n)$ time. Different from our algorithm, their algorithm works even for $T=1$. However, their algorithm's complexity is always $\Omega(n)$ even when $d=O(1)$. The reason is that even when $d$ is small, although the approximation quickly becomes good, the algorithm does not know this, and has to wait for sufficient long before it can make sure. In comparison, our Count algorithm has $O\left(d^{3} \log ^{2} n\right)$ complexity, is deterministic, and outputs the exact $n$.

Researchers have also considered these distributed computing problems under other settings. Kuhn et al. [19] have studied CoNSENSUS, and its variants coordinated/simultaneous consensus, in the $T$-interval model without limit on message sizes. Under such a setting, results from [19] imply that all the problems considered in this paper can be solved in $O(d)$ rounds. Count has been studied in anonymous dynamic networks (e.g., [7, 17, 22-24, 26]), but all the algorithms there have $\Omega(n)$ complexity. Among these, similar to our algorithm, the design in [17] also aggregates all values to one node to solve Count. One of the major differences, however, is that they use random walks to do so, which relies on mixing time and results in $\tilde{O}\left(n^{5}\right)$ complexity. Our algorithm uses explicit aggregation paths, together with a range of other techniques, which eventually achieves $O\left(d^{3} \log ^{2} n\right)$ complexity. Some researchers (e.g., $\left.[8,10,28]\right)$ have studied LEADERELECT and CONSENSUS in directed dynamic networks, which is quite different from our undirected setting. Augustine et al. $[4,5]$ have studied LEADERELECT and CONSENSUs in dynamic network with node churn and where the topology is an expander. They rely on efficient random walks in expander graphs, which does not apply to our setting. Finally, there have been a body of works (e.g., [14]) on eventually-stable networks. The topology of an eventually-stable network may change from round to round, but such changes eventually stop and the algorithm should output sometime after that [12]. In comparison, our algorithms do not wait for the network to stop changing.

\section{OVERVIEW OF OUR COUNT ALGORITHM}

This section provides the key intuitions behind our CounT algorithm, and Section 3 gives the details. Section 4 presents our algorithms for MAX/MEdiAn/Sum/LEADERELECT/CONSENSUS/ CONFIRMEDFLOOD, all of which follow the same framework as our Count algorithm.

\subsection{Starting Point}

Let $\alpha$ be the largest id, among all the $n$ nodes in the network. Assume for now that all nodes know $\alpha$, and we remove this assumption later. In static networks, aggregation is known to be an efficient way to compute CounT: We first build a spanning tree rooted at node $\alpha$. Next each node contributes a value of 1 . These values are propagated upstream along the tree, while being aggregated (i.e., summed together) at intermediate tree nodes. Finally, node $\alpha$ gets the sum of all the values, and floods this Count result to all nodes.

In $T$-interval dynamic networks, however, the changing topology may disrupt the spanning tree. Namely, a tree edge may no longer exist when we need to use it, causing the value from some node $u$ (there can be many such $u$ 's) to get stuck somewhere in the middle of the tree path. Imagine that the entire tree aggregation process, including the building of the spanning tree, takes no more than $T$ rounds. By the $T$-interval model, there must exist some connected spanning subgraph that remains stable in those $T$ rounds. Recall that $H_{r}$ is the maximum such subgraph for round $r$ through $r+T-1$. Since $H_{r}$ is connected, there must exist some path from node $u$ to node $\alpha$ that persists throughout those $T$ rounds. But the problem is that the spanning tree may not contain that particular path hence the value from $u$ may get stuck in the tree. (If we could magically ensure that the spanning tree only uses edges in $H_{r}$, then all problems are solved.) Naively retrying with a different spanning tree does not lead to good complexity.

From the above simple scenario, it is also easy to see that while deleted edges cause problems, newly added edges (i.e., edges that did not exist before but are later created) do not immediately cause any harm. In fact, given that all edges in $H_{r}$ persist throughout all $T$ rounds, in those $T$ rounds the algorithm can temporarily ignore all edges that are newly added: Even after ignoring those newly added edges, there must still exist a path from every node $u$ to node $\alpha$. Hence the main challenge here is how to deal with deleted edges.

\subsection{Parallel Propagation Over All Paths}

Assume for now that the backbone diameter $d$ is known, and we remove this assumption later. (Recall that the CounT problem is still non-trivial even with known $d$.) Consider the set $L$ of all paths ${ }^{6}$

\footnotetext{
${ }^{6}$ Throughout this paper, we only need to be concerned with paths in a given (static) graph. In dynamic networks, sometimes researchers consider dynamic paths [21] we do not need those.
} 
from some node $u$ to node $\alpha$ with length at most $d$, in the topology of some round $r$. Let $l=|L|$, which can be exponentially large. Ignore for now the challenge of keeping track of all these paths. Our first key idea is to avoid committing to any specific path for propagating the value. Instead, node $u$ splits its value (of 1.0) into $l$ equal pieces, and propagates each piece along a different path, all in parallel and taking $O(d)$ rounds. We will imagine that a piece "moves" from one node to another along the path, which gives the standard mass conservation property $[3,16,17,29]$ - the sum of all these pieces on all nodes always remain fixed.

As the pieces are moving, it is possible for most of these $l$ paths to get cut by the adversary - this will cause most pieces to get stuck. But observe that the adversary can stall a piece only if a path existed when we computed the available paths, and then no longer exists when we actually use the path. We can thus effectively limit the adversary's damage by staggering the propagation and by spreading our stake. Specifically, node $u$ propagates its value of 1.0 over $x$ sequential intervals (we will set $x$ later), where each interval comprises of $O(d)$ rounds and only deals with a value of $\frac{1}{x}$. Within each interval, node $u$ further breaks the $\frac{1}{x}$ value into many pieces, with each piece corresponding to a distinct path that is still alive at the beginning of that interval. (Namely, if all $l$ paths are always alive in all $x$ intervals, then the value of 1.0 is split into total $x l$ pieces, with $l$ pieces for each interval.) A path cut by the adversary can then only affect a single interval.

Over the course of these $x$ intervals, the number of paths in $L$ that survive may gradually decrease, due to some edges being deleted. Assume that $T$ is no smaller than the total number of rounds in all these $x$ intervals. Then the number of surviving paths can decrease from at most $O\left(n^{d}\right)$ to at least 1 . It is "at least 1 " because by the definition of the backbone diameter $d$, the diameter of $H_{r}$ is at most $d$ and hence $H_{r}$ must contains a path from node $u$ to node $\alpha$ with length at most $d$. This path must have survived. Let $f_{i}$ be the fraction of paths that are alive (i.e., survive) at the end of interval $i$, among all the paths that were alive at the beginning of interval $i$. We then have $\prod_{i=1}^{x} f_{i} \geq \frac{1}{n^{d}}$. The sum of all those pieces that get stuck is at most $\sum_{i=1}^{x}\left(1-f_{i}\right) \cdot \frac{1}{x} \leq 1-\left(\frac{1}{n^{d}}\right)^{\frac{1}{x}}$, and the optimal strategy of the adversary is simply to have $f_{1}=f_{2}=\ldots=f_{x}$. With $x=d \log n$, the $\operatorname{sum} \sum_{i=1}^{x}\left(1-f_{i}\right) \cdot \frac{1}{x}$ will be at most $\frac{1}{2}$. This means that at least half of the value from each node $u$ will successfully reach node $\alpha$.

At the end of the $x$ intervals, the stuck pieces are simply left in various arbitrary locations across the network. To collect all these stuck pieces, we repeat the above entire process for $2 \log n$ times (called $2 \log n$ phases), where each phase has $x=d \log n$ intervals. (There is no need for $T$ to be larger than the total number of rounds in all phases $-T$ only needs to be larger than the number of rounds in a single phase.) When we repeat the above process, the nodes holding the stuck pieces will be viewed as new $u$ 's for that phase, except that each such $u$ will start with a value corresponding to the stuck pieces it is holding, instead of starting with a value of 1.0. With such a design, in each phase, node $\alpha$ collects at least half of the remaining value in the network. Since the total count is only $n$, after the $2 \log n$ phases, the leftover will be $n \cdot \frac{1}{n^{2}}=\frac{1}{n}<1$. Having node $\alpha$ round up the total collected value then gives the exact count $n$. Finally, note that the algorithm does not know $n$ beforehand, and hence cannot readily compute the quantities of $d \log n$ and $2 \log n$ - we deal with this later.

At this point, the above ideas can already enable us to solve Count in $O\left(d^{2} \log ^{2} n\right)$ time complexity: We need total $2 \log n$ phases, with each phase having $d \log n$ intervals and each interval having $O(d)$ rounds. The caveat is that we have made a number of significant assumptions along the way. Section 2.3 and 2.4 will explain how we remove all these assumptions, which entails a collection of non-trivial techniques. One of the techniques will add additional complexity to the algorithm, and our final Count algorithm will have $O\left(d^{3} \log ^{2} n\right)$ time complexity.

Before proceeding, we quickly stress that a proper balance between intervals and phases is needed for the idea to work. Without multiple phases, $\Theta\left(n^{2} d \log n\right)$ intervals would be needed for the leftover to be $\frac{1}{n}$. Without multiple intervals in each phase, $\Theta\left(n^{d} \log n\right)$ phases would be needed. Consider any node $u$ holding some pieces at the beginning of a phase. The key difference between intervals and phases is that for all intervals within that phase, these pieces all start their propagation from the same node $u$. This allows us to analyze based on the number of surviving paths from node $u$ to node $\alpha$. While in the next phase, these pieces may start (i.e., continue) their propagation from arbitrary locations in the network.

\subsection{Avoiding Excessive Bookkeeping}

We next overcome the challenge of keeping track of all the pieces and paths. Consider the simple example in Figure 1, where the network has a fixed topology, with $n=9$ and $d=4$. Here as shown in Figure 1(a), node $u_{1}$ has exactly 4 paths to node $\alpha$ with length at most $d$, out of which 3 paths have node $v$ as the second node on the path. Imagine that we split the value of 1.0 on node $u_{1}$ into 4 equal pieces, and then propagate one piece along each path. Obviously, instead of sending 3 individual pieces to node $v$ where each piece corresponds to $\frac{1}{4}$, node $u_{1}$ only needs to send to node $v$ a combined value of $\frac{3}{4}$. Similarly, as shown in Figure $1(\mathrm{~b})$, node $u_{2}$ has 3 paths to node $\alpha$ with length at most $d$, out of which 2 paths have node $v$ as the second node on the path. Hence node $u_{2}$ only needs to send to node $v$ a combined value of $\frac{2}{3}$ (corresponding to 2 pieces, each worth $\frac{1}{3}$ ). But now it is unclear what node $v$ should do: It gets 3 pieces from node $u_{1}$ and 2 pieces from node $u_{2}$, where different pieces correspond to different values. Each piece needs to follow its own respective path. Obviously, with exponential number of paths and pieces, this becomes tricky.

Allowing repeated nodes. Interestingly, we observe that allowing paths to contain repeated nodes (vertices) helps to overcome the above problem. From now on, we use simple paths (e.g., in Figure 1(a) through (b)) to refer to paths with no repeated nodes, while paths (e.g., in Figure 1(c) through (e)) in general may contain repeated nodes. To see why it helps to consider paths instead of simple paths, let us continue with the example in Figure 1. As shown in Figure 1(c), there are total 3 paths from node $v$ to node $\alpha$ with length at most $d-1$. Then it must hold, as illustrated in Figure 1(d), that there are exactly 3 paths of length at most $d$ going from node $u_{1}$ to node $\alpha$ with $v$ being the second node on the path. By the same reason, there also must be exactly 3 such paths from node $u_{2}$ to node $\alpha$ 


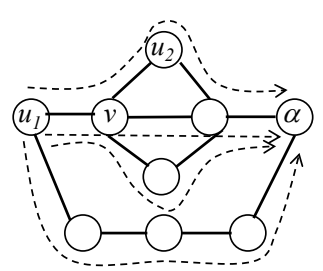

(a) There are total 4 simple paths from node $u_{1}$ to node $\alpha$ with length at most $d$. Among these, $\mathbf{3}$ simple paths have node $v$ as the second node on the simple path.

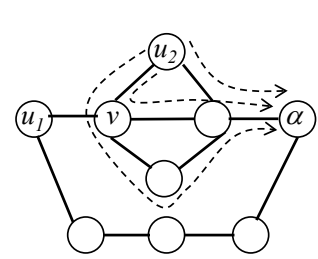

(b) There are total 3 simple paths from node $u_{2}$ to node $\alpha$ with length at most $d$. Among these, $\mathbf{2}$ simple paths have node $v$ as the second node on the simple path.

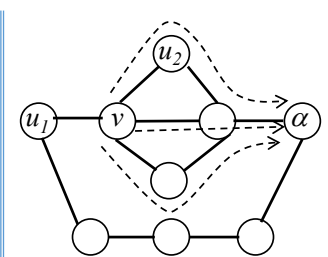

(c) There are total 3 paths from node $v$ to node $\alpha$ with length at most $d-1$. (In this example, all these paths happen to be simple paths.)

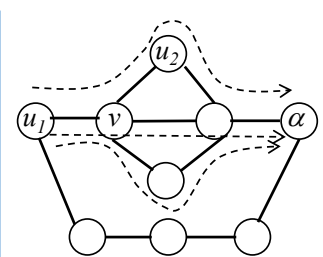

(d) There are total 3 paths from node $u_{l}$ to node $\alpha$ with length at most $d$, with node $v$ as the second node on the path. (In this example, all these paths happen to be simple paths.)

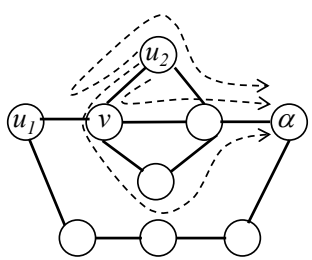

(e) There are total 3 paths from node $u_{2}$ to node $\alpha$ with length at most $d$, with node $v$ as the second node on the path. (In this example, one of the 3 paths is not a simple path.)

Figure 1: Why allowing repeated nodes on paths helps us to avoid excessive bookkeeping. This example has $d=4$.

(see Figure $1(\mathrm{e}))^{7}$ This means that node $v$ always gets exactly 3 pieces from each of its neighbors. For each set of 3 pieces, node $v$ should forward one piece along each of the 3 paths in Figure 1(c), regardless of which neighbor this set came from. Effectively, the pieces can now be processed in a memoryless way. In fact, it suffices for node $v$ to just add up (aggregate) all values it receives from all it neighbors, and send $\frac{1}{3}$ of the total value along each of the 3 paths in Figure 1(c).

A further optimization would enable node $v$ to do this by just sending a single message instead of 3 messages, and without node $v$ needing to know whether some of its previous neighbors no longer exist (i.e., due to edge removals). To do so, node $v$ simply sends the total value and the number 3. A neighbor $w$ of node $v$ simply takes a part of the value that is proportional to the number of paths going from node $w$ to node $\alpha$ with length at most $d-2$. At the same time, since node $v$ also receives a message from each of its current neighbors, node $v$ can simultaneously determine how much value has not been taken by its neighbors (due to edge removals). Such leftover value will be kept locally, to be fed into the next phase.

With the above ideas, now we only need to keep track of the number of paths going from each node to node $\alpha$. As a convenient consequence of using paths instead of simple paths, we will be able to easily count such paths efficiently via recursion. (Counting simple paths would instead be \#P-Complete.)

Rounding. In the above, when a node transfers a value to another node, precisely describing the value may take too many bits. To avoid this, we carefully round the value so that it takes $O(\log n)$ bits to encode. (We do not know $n$ and hence cannot compute $\log n$ - we deal with this later.) The discrepancy between the value and its rounded version will still be kept locally, to be fed into the next phase. (Not discarding the discrepancy is important.) We use similar rounding when counting the number of paths, and will show that such rounding does not compromise correctness.

\footnotetext{
${ }^{7}$ In comparison, recall that previously in Figure 1(a) and Figure 1(b), the corresponding numbers for simple paths were 3 and 2 for node $u_{1}$ and $u_{2}$, respectively.
}

\subsection{Dealing with Unknown $d, n$, and $\alpha$}

Unknown $d$. So far we have assumed the knowledge of the backbone diameter $d$ - recall that the algorithm should only use those paths with length at most $d$, and that the number of intervals in each phase needs to be $\Theta(d \log n)$. To remove this assumption, we use a standard doubling trick to guess $d$. Let $\tilde{d}$ be our current guess for $d$. The crux is to determine whether $\tilde{d}$ is too small.

To make such determination, we have node $\alpha$ distribute a predetermined number (i.e., $n^{\tilde{d}}$ ) of votes to all the nodes over $\tilde{d}$ rounds, where in each round each vote-holding node gives some of its votes to each of its neighbors. (We do not know $n-$ we deal with this later.) This value of $n \tilde{d}$ ensures that if $\tilde{d}$ is large enough (e.g., $\tilde{d} \geq d$ ), then every node will get at least one vote. Roughly speaking, this is because each node has at most $n$ neighbors, and the number of votes can only get "split" for $\tilde{d}$ times. On the other hand, if $\tilde{d}$ is too small, then some node will not get any vote. Those nodes then force all their neighbors to discard their respective votes. Since the topology is always connected, this causes the total number of remaining votes in the network to be less than $n^{\tilde{d}}$. We next invoke our earlier Count algorithm, for a second time, to count the remaining votes. (While the algorithm was for counting the number of nodes, it can be easily adapted to count votes, by using $\Theta(d \log n)$ instead of $\Theta(\log n)$ phases.) If $\tilde{d}$ is too small, we will find the vote count to be smaller than $n^{\tilde{d}}$.

Note that the approach may appear circular: When invoking the algorithm to count votes, we again need to know $d$. But it turns out that if $\tilde{d}$ is too small, our algorithm may undercount the votes but can never overcount. Hence if $\tilde{d}$ is too small, we will always find the vote count to be smaller than $n^{\tilde{d}}$.

Unknown $n$. While our goal is to compute $n$, our design so far has relied on the knowledge of $n$ for determining: (i) how many intervals/phases to run (Section 2.2), (ii) how many bits to use during rounding (Section 2.3), and (iii) how many votes to distribute (Section 2.4). One could try to potentially use randomization to first get some rough approximation of $n-$ but we aim for a (stronger) 
Table 1: Key notations.

\begin{tabular}{r|l}
\hline$n$ & total number of nodes \\
$d$ & backbone diameter \\
$T$ & parameter $T$ in $T$-interval dynamic network model \\
$\alpha$ & largest id among the $n$ nodes in the network \\
$\tilde{d}, \tilde{\alpha}$ & current guesses on $d$ and $\alpha$, respectively \\
$V$ & the set of all $n$ nodes in the network \\
$\sigma\left(r_{1}, r_{2}\right)$ & the maximum spanning subgraph contained \\
$\Gamma_{G}(u)$ & by all topologies from round $r_{1}$ to $r_{2}$ (both inclusive) \\
\hline
\end{tabular}

deterministic solution. To this end, we exploit the node ids: Instead of viewing the node ids as opaque, we view them as positive integers. Since i) there are total $n$ nodes, ii) all ids are unique, and iii) the length of each id is $O(\log n)$, we know that the largest id $\alpha$ among the $n$ nodes must satisfy $n \leq \alpha \leq \operatorname{poly}(n)$. This means that $\log n \leq$ $\log \alpha=\Theta(\log n)$. We thus could use $\log \alpha$ in place of $\log n$ for determining the parameters in the above three places, and we can show that doing so does not cause problems. A difficulty, however, is that the algorithm does not actually know $\alpha$. (If the algorithm were directly given a polynomial upper bound on $n$, there would be no such difficulty.) In fact, a trivial reduction shows that determining $\alpha$ is at least as hard as LEADERELECT, which is exactly one of the problems we aim to solve.

Unknown $\alpha$. We finally deal with the fact that the algorithm does not actually know $\alpha$. First, in the background, we let each node keep sending to its neighbors the largest id that it has seen so far (initially its own id). Next, for any given node $\tilde{\alpha}$, let $W$ be the set of nodes where $\tilde{\alpha}$ is the largest id they have seen so far. The nodes in $W$ will then together run an instance of our algorithm. Hence at any point of time, there may be multiple concurrent instances running. Each instance has its own root $\tilde{\alpha}$. When a node in an instance with smaller $\tilde{\alpha}$ learns about another instance with larger $\tilde{\alpha}$, the node switches to the latter instance. Switching into or out of an instance in the middle of its execution may cause various technical problems, and hence we only allow nodes to switch in/out at certain specific steps of an instance. We will show, via a careful analysis, that such "delayed" switches will not weaken the asymptotic properties. Finally, with the vote distribution/counting mechanism mentioned earlier, we will ensure that only the instance containing node $\alpha$ can output, when that instance has "grown" to include all nodes. We can then show that such output must be correct.

\section{OUR $O\left(d^{3} \log ^{2} n\right)$ COUNT ALGORITHM}

\subsection{Definitions and Technicalities}

Definitions. Table 1 summarizes our key notations. As a reminder, in our setting at the beginning of Section 1, we already defined $T$-interval dynamic network, backbone diameter, and time complexity. The following gives some more definitions.

Given a $T$-interval dynamic network, define $\sigma\left(r_{1}, r_{2}\right)$ to be the maximum (in terms of number of edges) spanning subgraph contained by all the topologies in all rounds from $r_{1}$ to $r_{2}$ (both inclusive). Given a node id $x$, we use " $x$ " to refer to the integer value of $x$, and "node $x$ " to refer to that node. Without loss of generality, we assume that node ids are never smaller than 2 . We use $V$ to denote the set of all nodes in the network, where $|V|=n$. We use $\alpha$ to denote the largest node id among the $n$ nodes in the network. Recall that $d$ is the backbone diameter of the dynamic network. We use $\tilde{d}$ and $\tilde{\alpha}$ to denote a node's current guesses on $d$ and $\alpha$, respectively. Without loss of generality, in each round, we add a self-loop to each node in the topology - hence every node is also a neighbor of itself, and receives its own message. For a given graph $G$ (with self-loops), a path of length $m$ is a sequence of nodes $x_{0}, x_{1}, \ldots, x_{m}$ such that $x_{i-1}$ is a neighbor of $x_{i}$ for all $i \in[1, m]$. In particular, a path may contain repeated nodes. With self-loops, we will only need to consider paths of length exactly $d$, rather than at most $d$. Let $\Gamma_{G}(u)$ be the eccentricity of node $u$ in $G$, and $\Gamma_{G}(u)=\infty$ if $G$ is disconnected. Recall from Section 2.4 that there may be multiple concurrent instances running in the network. We say that node $v$ does not interfere with instance $\tilde{\alpha}$ in round $r$ iff node $v$ in round $r$ only sends messages of the form $\langle x, y, \ldots\rangle$ where either $x \in\{$ SWITCH, OUTPUT $\}$ or $y \neq \tilde{\alpha}$.

Newcomer messages and oldcomer messages. In our algorithm, each node always sends a message in each round. Consider any given node $u$. Throughout our CounT algorithm (and across all the invocations of the various subroutines), node $u$ maintains a global variable $S$, which is initialized to the set of all positive integers. (Obviously, one can use finite data structures to achieve what we need for $S$ here.) At the end of each round, node $u$ receives a set of messages. A message whose sender's id is not in $S$ is a newcomer message, otherwise it is an oldcomer message. Unless otherwise mentioned in the algorithm, node $u$ will always ignore newcomer messages. After processing all the messages in this round, node $u$ immediately updates $S$ to be the ids of all the senders of the oldcomer messages received in this round. Finally, at the very beginning of each round, node $u$ has the option of resetting $S$ to the set of all positive integers, by invoking ResetNeighbors().

Intuitively, the above mechanism enables node $u$ to temporarily ignore messages coming from newly created (or newly recovered) edges in the network, until the next time that node $u$ invokes ResetNeighbors(). For clarity, our pseudo-codes do not explicit mention that newcomer messages are ignored. (We will, of course, clearly indicate when to invoke ResetNeighbors().) In several special places, our algorithm does not ignore newcomer messages, which we will clearly indicate.

Rounding. Section 2.3 mentioned that our algorithm sometimes (e.g., when a node transfers a value to another node) uses rounding to avoid needing more than $O(\log n)$ bits in each message. The following explains how exactly such rounding is done. Consider any real value $x \geq 0$, and consider the id $\tilde{\alpha}$ of any node in the network. (We must have $\tilde{\alpha} \leq \alpha \leq \operatorname{poly}(n)$.) When $x>0$, let the unique real value $a$ and integer $b$ be such that $1 \leq a<2$ and $x=a 2^{b}$. Our algorithm later will need to use both a "rounded-up" version of $x$ and a "rounded-down" version of $x$.

For the "rounded-up" version, our algorithm will be concerned with $x \in\left[1,2^{(n \tilde{\alpha})^{4}}\right]$. (The algorithm actually may also encounter the case of $x=0$. But obviously, this special case can be separately encoded using one extra bit.) For $x \in\left[1,2^{(n \tilde{\alpha})^{4}}\right]$, the corresponding $b$ value will be in $\left[0,(n \tilde{\alpha})^{4}\right]$, and can already be encoded using $O(\log n)$ bits. So we only need to properly round the $a$ value. To 


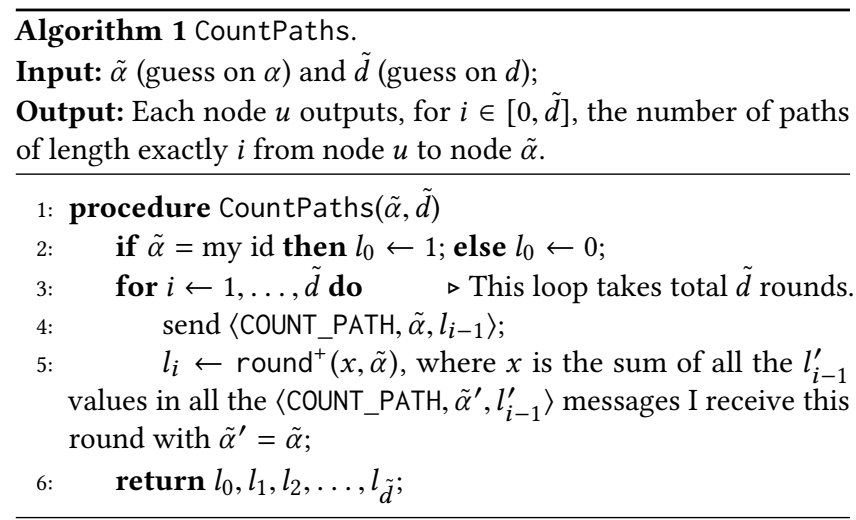

do so, we discretize $a$ using a granularity of $\frac{1}{\tilde{\alpha}^{6}}$. (We do not use a granularity of $1 / \operatorname{poly}(n)$ because the algorithm does not know $n$. In comparison, $\tilde{\alpha}$ will be explicitly maintained and hence known by the algorithm.) Specifically, let $a^{+}$be the smallest value such that $a^{+} \geq a$ and $a^{+}$is a multiple of $\frac{1}{\tilde{\alpha}^{6}}$. We define the "rounded-up" version of $x$ as $\operatorname{round}^{+}(x, \tilde{\alpha})=a^{+} \times 2^{b}$. For convenience, we also define $\operatorname{round}^{+}(0, \tilde{\alpha})=0$. Obviously, we have $x \leq \operatorname{round}^{+}(x, \tilde{\alpha}) \leq$ $\left(1+\frac{1}{\tilde{\alpha}^{6}}\right) x$. For all $x \in\left[1,2^{(n \tilde{\alpha})^{4}}\right]$, the value of $\operatorname{round}^{+}(x, \tilde{\alpha})$ can be encoded using $O(\log n)$ bits: To encode $\operatorname{round}^{+}(x, \tilde{\alpha})=a^{+} \times 2^{b}$, we only need to specify three integers $b, \tilde{\alpha}^{6}$, and $a^{+} \times \tilde{\alpha}^{6}$, taking only $O\left(\log \log 2^{(n \tilde{\alpha})^{4}}+\log \tilde{\alpha}\right)=O(\log n)$ bits.

For the "rounded-down" version, our algorithm will be concerned with $x \in\left[0,2^{(n \tilde{\alpha})^{4}}\right]$. We again use a granularity of $\frac{1}{\tilde{\alpha}^{6}}$. To avoid dealing with too small a $b$ value when $x$ is close to 0 , we define the "rounded-down" version of $x$ as $\operatorname{round}^{-}(x, \tilde{\alpha})=0$ for $x \in\left[0, \frac{1}{\tilde{\alpha}^{6}}\right)$. For $x \in\left[\frac{1}{\tilde{\alpha}^{6}}, 2^{(n \tilde{\alpha})^{4}}\right]$, we let $a^{-}$be the largest value such that $a^{-} \leq a$ and $a^{-}$is a multiple of $\frac{1}{\tilde{\alpha}^{6}}$, and we define the "rounded-down" version of $x$ as $\operatorname{round}^{-}(x, \tilde{\alpha})=a^{-} \times 2^{b}$. For all $x \in\left[0,2^{(n \tilde{\alpha})^{4}}\right]$, one can easily verify that $\left(1-\frac{1}{\tilde{\alpha}^{6}}\right) x-\frac{1}{\tilde{\alpha}^{6}} \leq \operatorname{round}^{-}(x, \tilde{\alpha}) \leq x$, and that the value of $\operatorname{round}^{-}(x, \tilde{\alpha})$ can be encoded using $O(\log n)$ bits.

\subsection{Building Blocks}

3.2.1 Counting paths. Algorithm 1 gives the subroutine for counting the number of distinct paths via a simple recursion. The time complexity of the algorithm will be much smaller than $T$, under our invocation parameters later and when $T \geq c d^{2} \log ^{2} n$. Line 5 of Algorithm 1 sets $l_{i} \leftarrow \operatorname{round}^{+}(x, \tilde{\alpha})$. Here rounding the value up (instead of down) ensures that during the value propagation later, a node never runs out of value to forward to the next hop. The network topology may change during the execution of Algorithm 1, potentially decreasing the number of paths as we go. This will not cause any problem, and no special mechanism is needed to avoid this. (Algorithm 1 never invokes ResetNeighbors(), hence the number of paths seen by the algorithm does not increase.)

3.2.2 Aggregating within an interval. Algorithm 2 gives the subroutine for aggregating the values, within an interval. Each interval here consists of $2 \tilde{d}$ rounds. Under invocation parameters later and when $T \geq c d^{2} \log ^{2} n$, the value $2 \tilde{d}$ will be much smaller than $T$. Recall from Section 2 that each node splits its value itv_input across all the paths, and propagates all those pieces to node $\tilde{\alpha}$ in parallel, with proper aggregation along the way. Algorithm 2 first invokes Algorithm 1 to count paths. For any node $u$, let $l_{i, u}$ be the computed number of paths from node $u$ to node $\tilde{\alpha}$ with length exactly $i$. Next the algorithm goes through $\tilde{d}$ steps. Intuitively, each step moves all pieces simultaneously one step further along their respective paths. Consider any node $u$ and any neighboring node $v$ of node $u$. In the first step, node $u$ sends a message containing the rounded-down version of its initial value itv_input. (We round down so that a node never sends more than what it has.) Doing so transfers $\frac{l_{\tilde{d}-1, v}}{l_{\tilde{d}, u}}$ fraction ${ }^{8}$ of $i t v \_i n p u t$ from node $u$ to node $v$ (and also transfers some other fractions from node $u$ to its other neighbors). This quantity should then be added to the value on node $v$ and subtracted from the value on node $u$. The remaining steps are similar: In the $i$-th step $(i \geq 2)$, each node sends its current local value, and we use the fraction $\frac{l_{\tilde{d}-i, v}}{l_{\tilde{d}-i+1, u}}$ instead of $\frac{l_{\tilde{d}-1, v}}{l_{\tilde{d}, u}}$.

3.2.3 Aggregating over multiple phases/intervals. Following the ideas in Section 2.2 and Section 2.4, Algorithm 3 gives the subroutine for summing up all the input parameters of all the invoking nodes. This is done over multiple phases, with each phase consisting of $\tilde{d} \log \tilde{\alpha}$ intervals. At the beginning of each phase, each node has some value remain. In each interval in that phase, the node invokes IntervalAggregate() with $\frac{\text { remain }}{\tilde{d} \log \tilde{\alpha}}$ being the input. Each such invocation will end up with some leftover value. The sum of all the leftover values from all these intervals will be fed into the next phase. Note that under our invocation parameters later and when $T \geq c d^{2} \log ^{2} n$, the total number of rounds in a phase will be no larger than $T$.

Later Algorithm 3 will be separately invoked for i) counting the number of nodes, and ii) counting the number of votes. For counting the number of votes, where the sum can be as large as $n^{d}$, Algorithm 3 is invoked with max_out $=O\left(n^{d}\right)$ and reset $=$ true. Having max_out $=O\left(n^{d}\right)$ results in $O(d \log n)$ phases, which in turn ensures the leftover value to be less than 1 even if the sum is as large as $n^{d}$. Having reset $=$ true causes Algorithm 3 to invoke ResetNeighbors() after each phase, enabling the algorithm to fully utilize those newly created edges in the network (see Section 3.1). For counting the number of nodes, Algorithm 3 is invoked with max_out $=O(n)$ and reset $=$ false. This gives $O(\log n)$ phases, without ResetNeighbors() being invoked after each phase. Here we do not want to invoke ResetNeighbors(), so that later Algorithm 4 can properly distribute votes as desired.

The following lemma summarizes the guarantees of Algorithm 3. The lemma's proof largely follows the intuition in Section 2.2, and is deferred to the full version [15] of this paper. For the lemma, recall that $V$ denotes the set of all nodes in the network. Also, recall that for a node id $x$, we use " $x$ " to refer to the integer value of $x$, and "node $x$ " to refer to that node.

LEMMA 3.1. Consider any round $r$, any node $\tilde{\alpha}$, any integer $\tilde{d}$ where $2 \leq \tilde{d} \leq \tilde{\alpha}$, any positive integer max_out, and any reset $\epsilon$

\footnotetext{
${ }^{8}$ If $l_{\tilde{d}, u}=0$, then node $u$ has no path of length $\tilde{d}$ to $\tilde{\alpha}$. This necessarily implies that node $v$ has no path of length $\tilde{d}-1$ to $\tilde{\alpha}$, and hence $l_{\tilde{d}-1, v}=0$ as well. In such a case, node $u$ will not transfer any value to node $v$. Hence we define $\frac{0}{0}=0$ here.
} 

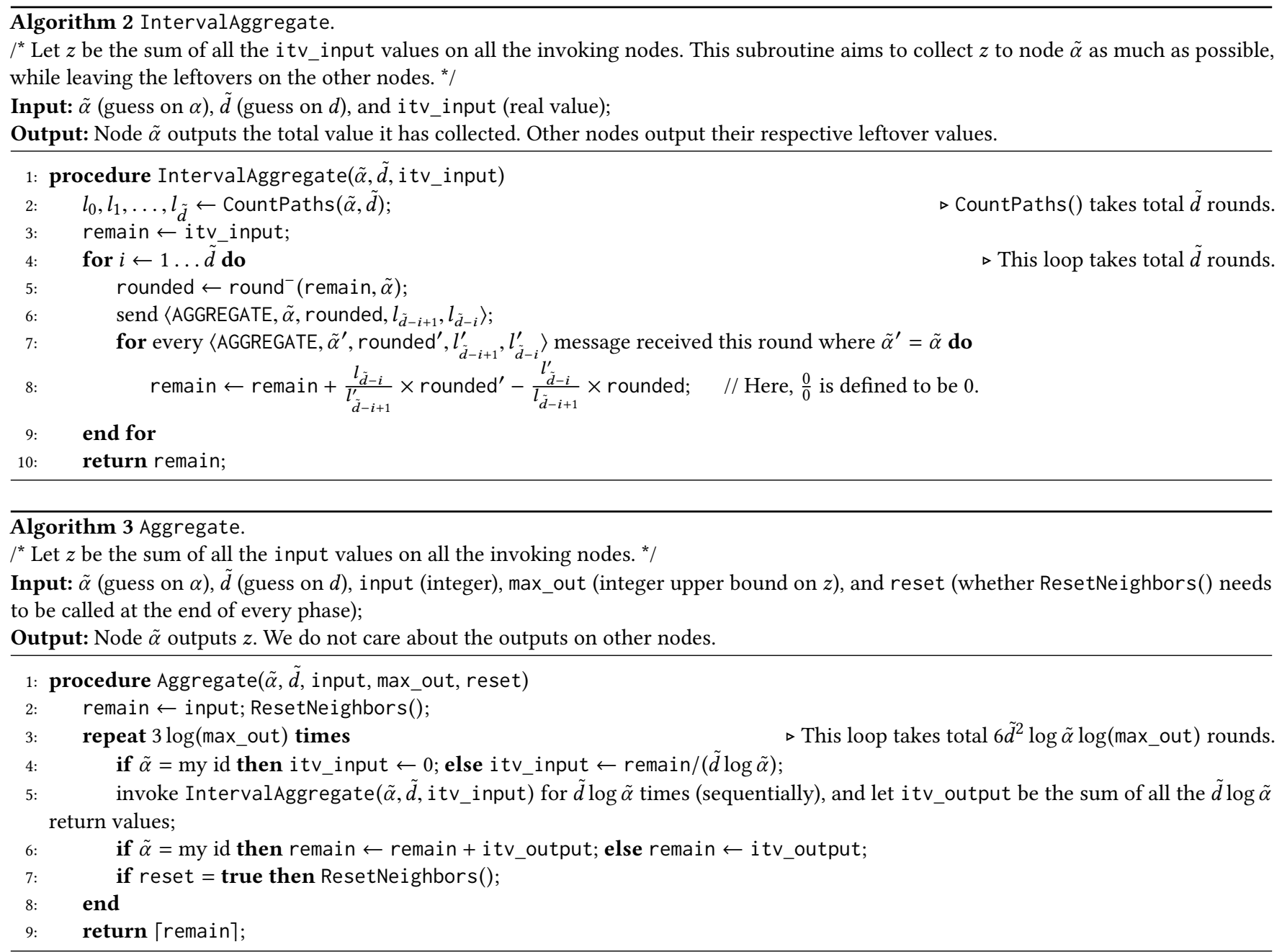

$\{$ true, false $\}$. Let $W$ be any set of nodes where node $\tilde{\alpha} \in W$ and where each node $u \in W$ invokes Aggregate $\left(\tilde{\alpha}, \tilde{d}\right.$, input $_{u}$, max_out, reset) (i.e., Algorithm 3) in round $r$ with some integer input $_{u} \geq 0$ such that $\sum_{u \in W}$ input $_{u} \leq n+\tilde{\alpha}^{\tilde{d}}$. Let output $\tilde{\alpha}$ be the return value of Algorithm 3 on node $\tilde{\alpha}$, and let $r^{\prime \prime}=r+6 \tilde{d}^{2} \log \tilde{\alpha} \log$ (max_out). If no node in $V \backslash W$ interferes with instance $\tilde{\alpha}$ in any round from round $r$ to round $r^{\prime \prime}-1$ (both inclusive), then we have:

$$
\text { output }_{\tilde{\alpha}} \leq \sum_{u \in W} \text { input }_{u}
$$

We further have:

$$
\text { output }_{\tilde{\alpha}}=\sum_{u \in W} \text { input }_{u},
$$

if all of the following four conditions hold:

- no node in $V \backslash W$ interferes with instance $\tilde{\alpha}$ in any round from round $r$ to round $r^{\prime \prime}-1$ (both inclusive);

- $W=V$ and $\tilde{\alpha} \geq n$;

- $\sum_{u \in W}$ input $_{u} \leq$ max_out;

- $\left(\right.$ reset $=$ false and $\tilde{d} \geq \Gamma_{G}(\tilde{\alpha})$, where $\left.G=\sigma\left(r, r^{\prime \prime}\right)\right)$ or (reset $=$ true, $\tilde{d} \geq d$, and $\left.T \geq 3 \tilde{d}^{2} \log \tilde{\alpha}\right)$.
Finally, for any $u \in W$, node u always sends $O(\log n)$ bits in every round of its execution of Algorithm 3.

3.2.4 Distributing votes. Following the ideas in Section 2.4, in order to check whether $\tilde{d}$ is sufficiently large, node $\tilde{\alpha}$ will distribute $\tilde{\alpha}^{\tilde{d}}$ votes to all the nodes, over about $\tilde{d}$ rounds. (By the definition of $\alpha$ and by the discussion in Section 2.4, we must have $\tilde{\alpha} \leq \alpha \leq$ poly $(n)$.) The quantity $\tilde{\alpha}^{\tilde{d}}$ ensures that each node, within distance of $\tilde{d}$ from node $\tilde{\alpha}$, gets at least one vote when $\tilde{\alpha} \geq n$. Algorithm 4 gives the subroutine for doing this. A simple design would be for each node, upon receiving some votes for the first time, to keep one vote for itself and distribute all the remaining votes to its neighbors. But this would need $\Theta(\tilde{d} \log \tilde{\alpha})$ message size. To reduce the message size to $O(\log n)$, in each round each node in Algorithm 4 only sends a single bit (in addition to also sending $\tilde{\alpha}$ ) indicating whether it has any votes. In the $i$-th iteration, if node $u$ has votes while a neighboring node $v$ has none, then exactly $\tilde{\alpha}^{\tilde{d}-i}$ votes are transferred from node $u$ to node $v$. Here the quantity $\tilde{\alpha}^{\tilde{d}-i}$ is implicit. We will show that when doing so, a node never runs out of votes to distribute, as long as it never has more than $\tilde{\alpha}$ neighbors. 


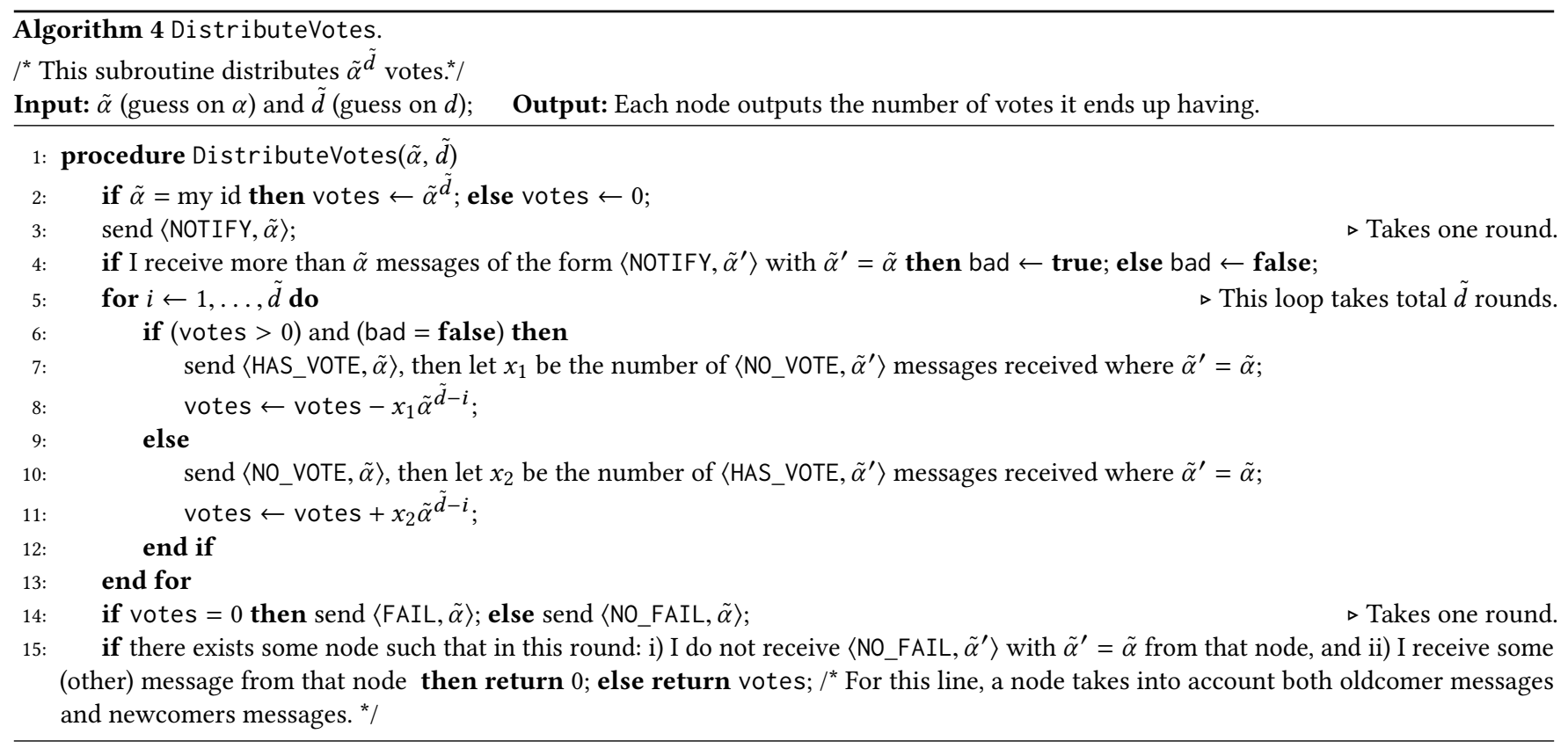

On the other hand, if the number of neighbors exceeds $\tilde{\alpha}$ (at Line 4), in Algorithm 4 the node simply refuses to distribute any vote, and will cause vote verification later to fail. This is intentional since more than $\tilde{\alpha}$ neighbors implies $\tilde{\alpha}<n$ and hence $\tilde{\alpha} \neq \alpha$. Failing the vote verification then forces the algorithm to later update $\tilde{\alpha}$. The following lemma (see proof in the full version [15] of this paper) summarizes the guarantees of Algorithm 4. (Also, recall that $V$ denotes the set of all nodes in the network.)

Lemma 3.2. Consider any rounds $r^{\prime}$ and $r$ where $r^{\prime} \leq r$, any node $\tilde{\alpha}$, and any integer $\tilde{d}$ where $2 \leq \tilde{d} \leq \tilde{\alpha}$. Let $W$ be any set of nodes that all (i) invoke DistributeVotes $(\tilde{\alpha}, \tilde{d})$ (i.e., Algorithm 4) simultaneously in round $r$, and (ii) last invoked ResetNeighbors() at the beginning of round $r^{\prime}$. Let $G_{1}=\sigma\left(r^{\prime}, r\right)$ and $G_{2}=\sigma\left(r^{\prime}, r+\tilde{d}+2\right)$. For $u \in W$, let output ${ }_{u}$ be the return value of Algorithm 4 on node $u$. If (i) $\tilde{\alpha} \in W$, and (ii) for all $v \in V \backslash W$ and in every round from round $r$ to round $r+\tilde{d}+1$ (both inclusive), node $v$ sends some message but does not interfere with instance $\tilde{\alpha}$, then all the following holds:

- $\sum_{u \in W}$ output $_{u} \leq \tilde{\alpha}^{\tilde{d}}$ and output ${ }_{u}$ is a non-negative integer for all $u \in W$.

- If $\sum_{u \in W}$ output $_{u}=\tilde{\alpha} \tilde{d}$, then $W=V$ and $\tilde{d} \geq \Gamma_{G_{1}}(\tilde{\alpha})$.

- If $W=V, \tilde{d} \geq \Gamma_{G_{2}}(\tilde{\alpha})$, and $\tilde{\alpha} \geq n$, then $\sum_{u \in W}$ output $_{u}=$ $\tilde{\alpha}^{\tilde{d}}$.

Finally, for all $u \in W$, node $u$ always sends $O(\log n)$ bits in every round of its execution of Algorithm 4.

\subsection{Putting Everything Together}

Algorithm 5 gives our final algorithm for Count, which follows the intuition in Section 2.4. Namely, the nodes first invoke Aggregate() to count the number of nodes. Next they use DistributeVotes() to distribute $\tilde{\alpha}^{\tilde{d}}$ votes, and then invoke Aggregate() again to count the total number of votes. If the second Aggregate() result matches $\tilde{\alpha} \tilde{d}$, the nodes output.

In Algorithm 5, each node runs CountNodes() and FloodRoot() in parallel. In FloodRoot(), each node keeps sending the largest id that it has seen so far. If this id equals its own id, then the node will initiate a new "instance", by flooding a SYNC message. Multiple nodes may start flooding such SYNC messages simultaneously. Other nodes will wait until they receive the first such SYNC message, and join the "instance" corresponding to that SYNC message. The SYNC message also carries information on when this "instance" should start - this enables all nodes in the "instance" to invoke Aggregate() and DistributeVotes() in a synchronized fashion (at Line 14 to 16). A node may need to switch from one "instance" to another. To avoid various technical issues, we do not allow such switch to happen during the invocations of Aggregate() and DistributeVotes(), and hence the switch may be delayed. Finally, all the SWITCH/SYNC/OUTPUT messages in the algorithm are "signaling" messages, and we want nodes to process them as soon as possible. Hence a node will process (rather than ignore) these messages even if they are newcomer messages.

With FloodRoot(), all nodes will see the largest id $\alpha$ (among the $n$ nodes) within $d$ rounds. All nodes hence will later switch into the "instance" whose root is node $\alpha$, which eventually causes the algorithm to produce a correct answer. A careful reasoning will show that the delayed switches will not blow up the time complexity. The following theorem summarizes the final guarantees of our Count algorithm, with proof in the full version [15] of this paper:

THEOREM 3.3. There exists some constant $c$ independent of $d, n$, and $T$, such that as long as $T \geq c d^{2} \log ^{2} n$ :

- Algorithm 5 always outputs $n$ (and terminates) in $O\left(d^{3} \log ^{2} n\right)$ rounds.

- In each round during the execution of Algorithm 5, each node sends only $O(\log n)$ bits. 


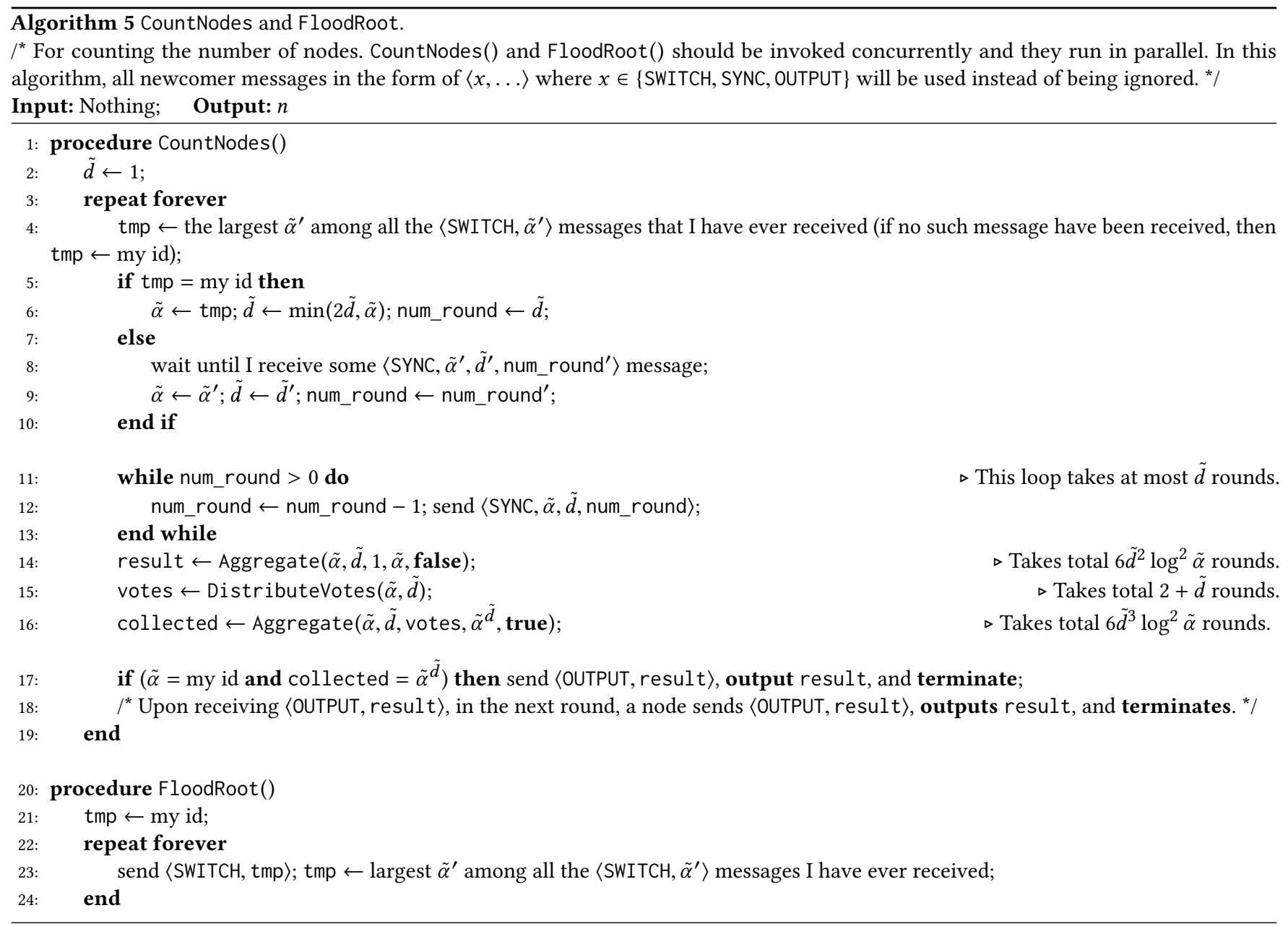

\section{OUR $O\left(d^{3} \operatorname{polylog}(n)\right)$ ALGORITHMS FOR OTHER PROBLEMS}

We have presented our $O\left(d^{3} \log ^{2} n\right)$ Count algorithm. The general framework in this CounT algorithm can be adapted to solve a range of other problems, as following, when $T \geq c d^{2} \log ^{2} n$. For solving MAX/LEAdERElect/ CONSENSUS/CONFIRMEdFlood, we only need to replace Line 14 in Algorithm 5 in the following way. Instead of invoking Aggregate(), Line 14 will now simply flood, for $2 \tilde{d}$ rounds, the maximum input value seen (for MAX and Consensus) or the maximum node id seen (for LEADERELECT) or the input of the distinguished node (for CONFIRMEDFLOOD). When the condition at Line 17 is satisfied, $\tilde{d}$ must have been large enough, and hence every node must have previously in Line 14 "heard from" all nodes. This then enables the algorithm to output a correct result in $O\left(d^{3} \log ^{2} n\right)$ rounds.

For solving Sum and Median, we first invoke the Count algorithm to obtain $n$. Note that when the algorithm terminates, all nodes must already see the largest id $\alpha$ among all the $n$ nodes. Hence in all future invocations of the algorithm, there will only be a single "instance" - namely, the "instance" whose root is $\alpha$. Next, we invoke the algorithm again to get the maximum value $z$ among the $n$ input values. Finally, for Sum, we invoke the algorithm a third time while changing Line 14 from "result $\leftarrow \operatorname{Aggregate}(\tilde{\alpha}, \tilde{d}, 1, \tilde{\alpha}$, false)" to "result $\leftarrow$ Aggregate $(\tilde{\alpha}, \tilde{d}, x, n z$, false)", with $x$ being the local input on the node. Doing so solves Sum in $O\left(d^{3} \log ^{2} n\right)$ rounds. For MEdiAn, after getting $n$ and $z$, node $\alpha$ does a binary search in the range of $[0, z]$. In each step of the search, node $\alpha$ indicates the current range of interest, and uses the algorithm to count the number of inputs falling within that range. This solves MEDIAN in $O\left(d^{3} \log ^{3} n\right)$ rounds.

\section{ACKNOWLEDGMENTS}

We thank the anonymous SPAA reviewers for their helpful feedbacks on this paper. This work is partly supported by the research grant MOE2017-T2-2-031 from Singapore Ministry of Education Academic Research Fund Tier-2.

\section{REFERENCES}

[1] Sebastian Abshoff, Markus Benter, Andreas Cord-Landwehr, Manuel Malatyali, and Friedhelm Meyer auf der Heide. 2013. Token Dissemination in Geometric Dynamic Networks. In ALGOSENSORS.

[2] M. Ahmadi and F. Kuhn. 2017. Multi-message Broadcast in Dynamic Radio Networks. In ALGOSENSORS. 
[3] P. Almeida, C. Baquero, M. Farach-Colton, Jesus O, and Mosteiro N. 2017. Faulttolerant aggregation: Flow-Updating meets Mass-Distribution. Distributed Computing 30, 4 (2017), 281-291.

[4] John Augustine, Gopal Pandurangan, and Peter Robinson. 2013. Fast byzantine agreement in dynamic networks. In PODC.

[5] J. Augustine, G. Pandurangan, and P. Robinson. 2015. Fast Byzantine Leader Election in Dynamic Networks. In DISC.

[6] P. Brandes and F. Meyer auf der Heide. 2012. Distributed Computing in Faultprone Dynamic Networks. In International Workshop on Theoretical Aspects of Dynamic Distributed Systems.

[7] M. Chakraborty, A. Milani, and M. Mosteiro. 2018. A Faster Exact-Counting Protocol for Anonymous Dynamic Networks. Algorithmica 80, 11 (Nov 2018), 3023-3049.

[8] Bernadette Charron-Bost, Matthias Fugger, and Thomas Nowak. 2015. Approximate Consensus in Highly Dynamic Networks: The Role of Averaging Algorithms. In ICALP.

[9] Binbin Chen, Haifeng Yu, Yuda Zhao, and Phillip B. Gibbons. 2014. The Cost of Fault Tolerance in Multi-Party Communication Complexity. F. ACM 61, 3 (May 2014), 19:1-19:64.

[10] E. Coulouma and E. Godard. 2013. A Characterization of Dynamic Networks where Consensus is Solvable. In SIROCCO.

[11] Atish Das Sarma, Anisur Molla, and Gopal Pandurangan. 2012. Fast Distributed Computation in Dynamic Networks via Random Walks. In DISC.

[12] S. Dolev. 2000. Self-Stabilization. MIT Press.

[13] Bernhard Haeupler and David Karger. 2011. Faster information dissemination in dynamic networks via network coding. In PODC.

[14] R. Ingram, P. Shields, and J. Walter. 2009. An asynchronous leader election algorithm for dynamic networks. In IPDPS.

[15] Irvan Jahja and Haifeng Yu. 2020. Sublinear Algorithms in Tinterval Dynamic Networks. Technical Report. School of Computing, National University of Singapore. Also available at https://www. comp. nus.edu.sg/\%7Eyuhf/pathcount-spaa20-tr.pdf.
[16] D. Kempe, A. Dobra, and J. Gehrke. 2003. Gossip-Based Computation of Aggregate Information. In FOCS.

[17] D. Kowalski and M. Mosteiro. 2018. Polynomial Counting in Anonymous Dynamic Networks with Applications to Anonymous Dynamic Algebraic Computations. In ICALP.

[18] Fabian Kuhn, Nancy Lynch, and Rotem Oshman. 2010. Distributed Computation in Dynamic Networks. In STOC.

[19] Fabian Kuhn, Yoram Moses, and Rotem Oshman. 2011. Coordinated Consensus in Dynamic Networks. In PODC.

[20] Fabian Kuhn and Rotem Oshman. 2011. The complexity of data aggregation in directed networks. In DISC.

[21] Fabian Kuhn and Rotem Oshman. 2011. Dynamic networks: Models and algorithms. SIGACT News 42, 1 (March 2011), 82-96.

[22] G. Luna, R. Baldoni, S. Bonomi, and I. Chatzigiannakis. 2014. Conscious and unconscious counting on anonymous dynamic networks. In International Conference on Distributed Computing and Networking.

[23] G. Luna, R. Baldoni, S. Bonomi, and I. Chatzigiannakis. 2014. Counting in Anonymous Dynamic Networks Under Worst-Case Adversary. In IEEE International Conference on Distributed Computing Systems.

[24] G. Luna, S. Bonomi, I. Chatzigiannakis, and R. Baldoni. 2013. Counting in anonymous dynamic networks: An experimental perspective. In ALGOSENSORS.

[25] Nancy Lynch. 1996. Distributed Algorithms. Morgan Kaufmann.

[26] O. Michail, I. Chatzigiannakis, and P. Spirakis. 2013. Naming and Counting in Anonymous Unknown Dynamic Networks. In Proceedings of International Symposium on Stabilization, Safety, and Security of Distributed Systems.

[27] D. Peleg. 1987. Distributed Computing: A Locality-Sensitive Approach. Society for Industrial and Applied Mathematics.

[28] U. Schmid, B. Weiss, and I. Keidar. 2009. Impossibility Results and Lower Bounds for Consensus Under Link Failures. SIAM 7. Comput. 38, 5 (2009), 1912-1951.

[29] Wesley W Terpstra, Christof Leng, and Alejandro P Buchmann. 2007. Brief Announcement: Practical summation via gossip. In PODC.

[30] H. Yu, Y. Zhao, and I. Jahja. 2018. The Cost of Unknown Diameter in Dynamic Networks. J. ACM 65, 5 (Sept. 2018), 31:1-31:34 